\title{
Mechanisms of in vitro development of resistance to metronidazole in Trichomonas vaginalis
}

\author{
Dominique Rasoloson, Štěpánka Vaňáčová, Eva Tomková, Jakub Rázga, \\ Ivan Hrdý, Jan Tachezy and Jaroslav Kulda
}

Department of Parasitology, Faculty of Science, Charles University in Prague, Viničná 7, 12844 Prague 2, Czech Republic
Author for correspondence: Jaroslav Kulda. Tel: +42 022195 3206. Fax: +420224919704. e-mail:kulda@natur.cuni.cz

Development of resistance against metronidazole and mechanisms responsible for this process were studied in a sexually transmitted pathogen of humans, Trichomonas vaginalis. Monitoring of changes in metabolism and protein expression that accompanied increasing resistance of strains derived from a common drug-susceptible parent (TV 10-02) showed the multistep character of the process. The aerobic type of resistance known to occur in isolates from patients non-responsive to treatment appeared at the earliest stage, followed by development of the anaerobic type of resistance which was accompanied by gradual loss of hydrogenosomal proteins associated with drug-activating pathways [pyruvate: ferredoxin oxidoreductase (PFOR), hydrogenase, ferredoxin]. Unexpectedly, the loss of PFOR did not result in acquisition of full anaerobic resistance, thus indicating an alternative source of electrons required for the drug activation. These data suggest involvement of the oxidative decarboxylation of malate in hydrogenosomes, catalysed by NAD ${ }^{+}$. dependent malic enzyme and subsequent transfer of reduced equivalents to the drug via NADH: ferredoxin oxidoreductase and ferredoxin. Accordingly, all components of this pathway were eliminated before the resistance was fully developed. Resistant Trichomonas vaginalis compensated the impaired function of hydrogenosomes by enhanced conversion of pyruvate to lactate in the cytosol. Further analysis of the two key enzymes involved in metronidazole activation by Northern blotting and assay for nascent mRNA showed that the insufficient expression of the PFOR protein results from decreased gene transcription, while down-regulation of malic enzyme is controlled at the mRNA level.

Keywords: drug resistance, hydrogenosome, pyruvate: ferredoxin oxidoreductase, ferredoxin, hydrogenosomal malic enzyme

\section{INTRODUCTION}

Metronidazole and related derivatives of 5-nitroimidazole are potent drugs against infections with anaerobic or microaerophilic protozoa and bacteria. The antimicrobial activity of these drugs is selective, depending on metabolic activation of the drug within the target

\footnotetext{
Abbreviations: EPR, electron paramagnetic resonance; $L D H$, lactate dehydrogenase; ME, malic enzyme; MLC, minimal lethal concentration; NADH:FOR, NADH:ferredoxin oxidoreductase; PFOR, pyruvate:ferredoxin oxidoreductase; STK, succinyl thiokinase.
}

cell, resulting in the release of reactive nitro anion radicals. A common property of susceptible microorganisms is the presence of electron generating and transport systems of low redox potential, able to mediate reduction of the drug (Edwards, 1993; Müller, 1986). In Trichomonas vaginalis, the protozoal agent of a sexually transmitted disease of the human urogenital tract, activation of 5-nitroimidazoles takes place in an organellar compartment, the hydrogenosome (Müller, 1993). Electrons required for the drug reduction are generated by the key hydrogenosomal enzyme pyruvate:ferredoxin oxidoreductase (PFOR; EC 1.2.7.1) catalysing oxidative decarboxylation of pyruvate to acetyl-CoA 
and $\mathrm{CO}_{2}$. The pathway is coupled to ATP production at the substrate level and its final product is acetate. Electrons released in the PFOR reaction are accepted by ferredoxin that is subsequently reoxidized by hydrogenase (EC 1.18.3.1). In this reaction electrons are coupled to protons to form molecular hydrogen, another final product of hydrogenosomal metabolism (Lindmark \& Müller, 1973). If metronidazole is present it acts as a preferential electron acceptor, effectively competing for electrons with the hydrogenase. Consequently, activation of the drug is reflected in reduced hydrogen production by the organelle (Lloyd \& Kristensen, 1985). Direct evidence for metronidazole anion radicals release in pyruvate-stimulated hydrogenosomes has been provided for Trichomonas vaginalis (Chapman et al., 1985), as well as for the related pathogen of cattle, Tritrichomonas foetus, (Moreno et al., 1983) by using electron paramagnetic resonance (EPR) spectroscopy. Another ferredoxin-linked oxidoreductase reaction in hydrogenosomes is oxidative decarboxylation of malate to pyruvate, catalysed by the hydrogenosomal malic enzyme (ME; EC 1.1.1.39) (Drmota et al., 1996). The reaction is $\mathrm{NAD}^{+}$-dependent, the $\mathrm{NADH}$ produced is reoxidized by the $\mathrm{NADH}$ :ferredoxin oxidoreductase (NADH:FOR; EC 1.18.1.3) and electrons are transferred to ferredoxin. The pathway is bi-directional, able to proceed toward malate production as shown by experiments with isolated organelles (Steinbüchel \& Müller, 1986). The potential of this pathway for metronidazole reduction has not been taken into consideration.

Resistance to metronidazole and related 5-nitroimidazole drugs has been demonstrated both in field isolates of Trichomonas vaginalis from patients refractory to treatment (Kulda et al., 1982; Lossick et al., 1986; Meingassner \& Thurner, 1979; Meri et al., 2000; Müller et al., 1980) and in laboratory-developed strains obtained by exposing trichomonads to sublethal pressure of the drug either in vitro (Brown et al., 1999; Kulda et al., 1984, 1993; Tachezy et al., 1993) or in vivo (de Carneri et al., 1969; Meingassner et al., 1978). Two distinct types of resistance have been recognized and named aerobic and anaerobic according to conditions required for demonstration of resistance by susceptibility assays in vitro (Kulda, 1999). The aerobic resistance typically occurs in isolates from treatmentrefractory patients. It is manifested only if some oxygen is present. Under anaerobiosis the resistant organisms succumb to the drug, as they possess a functional drugactivating pathway. The resistance apparently results from defective oxygen scavenging and subsequent interference of intracellular oxygen with the drug activation (Ellis et al., 1992; Rasoloson et al., 2001; Yarlett et al., 1986a). Alternatively, defective redox properties of ferredoxin or decreased ferredoxin levels (Yarlett et al., 1986b) due to altered transcription of the ferredoxin gene (Quon et al., 1992) have been proposed as potential mechanisms. However, a causal relationship of these alterations with the aerobically resistant phenotype has not been convincingly demonstrated. The anaerobic resistance resides in elimination of pathways responsible for the reductive activation of the drug. So far it has been demonstrated only in laboratory-developed strains (Brown et al., 1999; Kulda et al., 1984, 1993). It is detectable under anaerobic conditions and characterized by very high minimum lethal concentration (MLC) values in vitro (over $1000 \mu \mathrm{g}$ metronidazole $\mathrm{ml}^{-1}$ ). This type of resistance has been demonstrated in Tritrichomonas foetus (Čerkasovová et al., 1984; Kulda et al., 1984). The loss of PFOR activity holds for a key attribute of the anaerobic resistance. As shown by monitoring of the in vitro development of resistance in a Tritrichomonas foetus clone (Kabícková et al., 1987), the increasing resistance was strictly paralleled by the decrease of PFOR activity and uptake of the radiolabelled drug (Kulda et al., 1989), indicating the progressive failure of the parasite to metabolize the drug. Surprisingly, preliminary experiments with Trichomonas vaginalis (Čerkasovová et al., 1987) suggested that the development of anaerobic resistance in the human trichomonad does not follow the expected pattern. In vitro-obtained lines deficient in PFOR activity showed only low levels of anaerobic resistance to metronidazole, thus indicating involvement of an additional pathway which can provide electrons for the drug reduction.

In this study we report on changes accompanying development of resistance to metronidazole in a Trichomonas vaginalis strain exposed to increasing pressure of the drug in vitro. We monitored enzyme activities and metabolic end products of trichomonads at various stages of resistance development and investigated their correlation with the resistance phenotype of parasites. In a further step we followed expression of key hydrogenosomal proteins involved in drug activation and resistance, and tried to reveal by nucleic acid analysis at which level their expression is regulated. Preliminary results were reported at the following meetings: 2nd European Congress of Protistology, Clermont-Ferrand, France, 1995; 10th International Congress of Protozoology, Sydney, Australia, 1997; 3rd COST-B9 Meeting on Antiprotozoal Chemotherapy, Bruges, Belgium, 2000; and in a review (Kulda, 1999).

\section{METHODS}

Strains of parasites and resistance development. Trichomonas vaginalis strain TV 10-02, susceptible to metronidazole, and five drug-resistant derivatives of this strain (MR-3, MR-5, MR-30, MR-50, MR-100) were used in this study. The wildtype strain, TV 10-02, was isolated from a female patient examined at the University Hospital in Prague (Tachezy et al., 1993). The symptomatic infection of this patient was successfully cured with a single course of metronidazole and drug susceptibility of the isolated strain was confirmed by an in vitro assay. The metronidazole-resistant strains were developed by prolonged cultivation of the parent strain under increasing pressure of the drug, as described previously (Kulda et al., 1993; Tachezy et al., 1993). At different time intervals the cells were collected and cryopreserved. Cultures retrieved from these sequential stabilates served for monitoring pheno- 
Table 1. Histories of metronidazole-resistant strains derived in vitro from the drug-susceptible Trichomonas vaginalis strain TV 10-02

Trichomonads were maintained in a complete TYM medium at given concentrations of the drug by serial transfers in $48 \mathrm{~h}$ intervals. Metronidazole concentrations are given in $\mu \mathrm{g} \mathrm{ml}^{-1}$.

\begin{tabular}{|c|c|c|c|c|c|c|c|c|c|c|c|c|}
\hline \multirow[t]{2}{*}{ Strain } & & \multicolumn{11}{|c|}{ Time of exposure to metronidazole (days) } \\
\hline & Metronidazole concn: & 1 & 2 & 3 & 5 & 10 & 20 & 30 & 50 & 60 & 80 & 100 \\
\hline MR-3 & & - & - & 50 & & & & & & & & \\
\hline MR-5 & & - & - & 80 & 50 & & & & & & & \\
\hline MR-30 & & 41 & 28 & - & 36 & 44 & 112 & 85 & & & & \\
\hline MR-50 & & 41 & 28 & - & 36 & 44 & 4 & 28 & 245 & & & \\
\hline MR-100 & & 41 & 28 & - & 36 & 44 & 4 & 138 & 40 & 8 & 20 & 286 \\
\hline
\end{tabular}

typic changes accompanying the resistance development. Histories of the resistant strains employed in this study are listed in Table 1.

Trichomonads were grown at $37^{\circ} \mathrm{C}$ in Diamond's Trypticase-Yeast Extract-Maltose (TYM) medium (Diamond, 1957) supplemented with $10 \%$ heat-inactivated horse serum. All strains were cryopreserved in the presence of $5 \%$ dimethylsulfoxide $(\mathrm{v} / \mathrm{v})$ shortly after isolation and stored in liquid nitrogen until used in experiments. Active stock cultures of the resistant strains were maintained in a complete TYM medium with metronidazole $\left(3,5\right.$ or $30 \mu \mathrm{g} \mathrm{ml}^{-1}$ for MR-3, MR-5 and MR-30, respectively, and $50 \mu \mathrm{g} \mathrm{ml} \mathrm{l}^{-1}$ for MR-50 and MR-100). The ability of the MR-100 strain to grow at $100 \mu \mathrm{g}$ metronidazole $\mathrm{ml}^{-1}$ was rechecked before experiments. In final passages before harvesting, the cells were grown in medium without agar and metronidazole.

Susceptibility assays. Susceptibility of trichomonads to metronidazole was determined in vitro by using a microtitre plate assay as described previously (Tachezy et al., 1993). Trichomonads suspended in TYM medium without agar were exposed to twofold serial dilutions of metronidazole ranging from 1600 to $0.78 \mu \mathrm{g} \mathrm{m} l^{-1}$ and incubated at $37^{\circ} \mathrm{C}$ in air in a humid chamber (aerobic test) or in an anaerobic jar containing $\mathrm{H}_{2}$ with $5 \% \mathrm{CO}_{2}$ (anaerobic test). Aerobic assays with strains which did not tolerate exposure to air were done under an atmosphere containing $5 \% \mathrm{O}_{2}$ in a mixture of $5 \% \mathrm{CO}_{2}$ and $95 \% \mathrm{~N}_{2}$. After $48 \mathrm{~h}$ incubation, the plates were examined with an inverted microscope to check for motility of trichomonads. MLC was defined as the lowest concentration of the drug at which no motile trichomonads were observed. The end points were confirmed by failure of non-motile trichomonads to grow after reinoculation into drug-free medium.

Cell fractionation. The cells were harvested by centrifugation, washed three times in PBS $(\mathrm{pH} 7 \cdot 4)$ and resuspended in the isolation medium (Čerkasov et al., 1978) supplemented with leupeptin $\left(10 \mu \mathrm{g} \mathrm{ml}^{-1}\right)$, tosyl-lysine chloromethyl ketone $(1 \mathrm{mM})$ and dithiothreitol $(10 \mathrm{mM})$. The cell suspension was homogenized by sonication at $10 \mathrm{~W}$ for 3 cycles of $5 \times 1 \mathrm{~s}$ on ice. Cytosolic and large-granule fractions rich in hydrogenosomes were isolated from the cell homogenate by differential centrifugation as described by Cerkasov et al. (1978). For some experiments hydrogenosomes were purified on a Percoll gradient according to Lahti et al. (1992). The intactness of hydrogenosomes was assesed by measurement of at least $90 \%$ ME latency (Drmota et al., 1996) immediately after the isolation of the organelles.
Enzyme assays. Enzyme activities were assayed spectrophotometrically at $25^{\circ} \mathrm{C}$ under aerobic conditions or in anaerobic cuvettes under an atmosphere of $\mathrm{H}_{2}$ or $\mathrm{N}_{2}$. Certified oxygenfree nitrogen (99.999\% purity) passed through an Oxiclear column (Labclear) was employed. The large-granule fraction rich in hydrogenosomes was used for monitoring activities of the hydrogenosomal enzymes PFOR, hydrogenase, $\mathrm{NADH}$ :FOR and $\mathrm{NAD}^{+}$-dependent ME. Enzymic activities of lactate dehydrogenase $(\mathrm{LDH})$, pyruvate kinase and $\mathrm{NADP}^{+}$dependent ME were measured in the cytosolic fraction. Activities of PFOR, hydrogenase and NADH:FOR were determined under anaerobiosis at $600 \mathrm{~nm}$ as the rate of reduction of methyl viologen. The assays were performed using pyruvate and $\mathrm{H}_{2}$ as substrates for PFOR and hydrogenase, respectively (Kabíčková et al., 1987); NADH served as substrate for NADH:FOR (Thong \& Coombs, 1987). The activities of MEs were determined under aerobic conditions at $340 \mathrm{~nm}$ as the rate of $\mathrm{NAD}^{+}$and $\mathrm{NADP}^{+}$reduction in the presence of malate for the hydrogenosomal or cytosolic enzyme, respectively (Drmota et al., 1996). Activity of LDH was determined at $340 \mathrm{~nm}$ as the rate of NADH oxidation (Bergmeyer, 1963). Activity of pyruvate kinase was monitored by the formation of ATP, using phosphopyruvate as a substrate (Mertens et al., 1992). Proteins were determined according to the Lowry method.

Determination of metabolic end products. Metabolic end products were determined by HPLC and GC. Trichomonads were resuspended in a simple isotonic medium (Doran, 1959), adjusted to $5 \times 10^{7}$ cells $\mathrm{ml}^{-1}$ and placed in $1 \mathrm{ml}$ aliquots into $3 \mathrm{ml}$ vials tightly closed with vaccine stoppers. The vials were flushed with nitrogen and incubated for $30 \mathrm{~min}$ at $37^{\circ} \mathrm{C}$. After incubation, cells were pelleted by centrifugation, the supernatant was filtered through Whatman membrane (PVDF $0.45 \mu \mathrm{m})$ and the metabolic products were determined by using a PL Hi-Plex $\mathrm{H}$ column conditioned with $5 \mathrm{mM} \mathrm{H}_{2} \mathrm{SO}_{4}$ and thermostatted at $65^{\circ} \mathrm{C}$. Organic acids and alcohols were

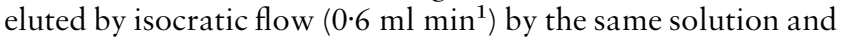
detected by UV at $205 \mathrm{~nm}$ (ECOM LCD 2082) and refractometry (Schodx Ri-71). The Chromatography Station for Windows (CSW) program was used for calculation of the product concentrations, according to calibration against standards. Glycerol concentration was calculated indirectly as the difference between refraction index and UV detector signals. Hydrogen production was determined by GC. Trichomonads were incubated as above for $60 \mathrm{~min}$, then the gas phase was analysed. The analysis was performed at room temperature using a molecular sieve $5 \mathrm{~A}$ column with nitrogen 
as carrier gas. Hydrogen was detected using a thermal conductivity detector with thermistors (Carlo Erba FractolabC). Each experiment was run in triplicate.

EPR spectroscopy. EPR spectroscopy was used for detection of nitro anion radicals released during activation of metronidazole by trichomonads. The cells were washed three times in $100 \mathrm{mM}$ phosphate buffer, $\mathrm{pH} 7 \cdot 5$, and resuspended in the same buffer with $40 \mathrm{mM}$ glucose to obtain a suspension with a density of $4 \times 10^{8}$ cells $\mathrm{ml}^{-1}$. The suspension was flushed with nitrogen to maintain anaerobic conditions. After the addition of $12 \mathrm{mM}$ metronidazole, $150 \mu \mathrm{l}$ of the suspension was immediately drawn into the EPR spectrometer cavity to measure the spectrum. Spectra were recorded on a Bruker ESP 300 (Bruker Spectrospin) at $20{ }^{\circ} \mathrm{C}$ using a quartz flat cellar liquid sample holder.

SDS-PAGE and Western blot analysis. Percoll-purified hydrogenosomes were used for the analysis of hydrogenosomal proteins. SDS-PAGE was performed on a Bio-Rad minislab gel apparatus using a 12 or $18 \%$ separating gel and a $5 \%$ stacking gel. Resolved proteins were stained by silver (Swain \& Ross, 1995) or blotted onto nitrocellulose membrane using a semi-dry transfer unit. The blots were allowed to react with polyclonal rabbit antibodies raised against hydrogenosomal ferredoxin, the $\alpha$-subunit of succinyl thiokinase (STK) (both provided by Patricia Johnson, UCLA, USA) and ME (Drmota et al., 1996), and an mAb against Trichomonas vaginalis PFOR (provided by Guy Brugerolle, University of Clermont Ferrand, France).

Preparation of DNA probes. Genomic DNA of Trichomonas vaginalis isolated by a modified guanidium thiocyanate method (Bowtell, 1987; Wang \& Wang, 1985a) was used as a template in a PCR amplification of specific DNA probes. The following primers were used: $\beta$-tubulin forward $5^{\prime}$-CATCGTCCCATCTCCAAAGG-3', reverse 5'-AATGGAACAAGGTTGACAGC-3'; ME forward 5'-AGGAAGAACGTGACCGCC-3', reverse 5'-GTTGCCGATATCGTGGTC-3'; PFOR forward 5'-GAYGGHACHGTNGGHGC-3', reverse 5'-TCRWADGCCCARCCRTC-3'; $16 \mathrm{~S}$ rRNA forward 5'-GGTGGTGCATGGCCG-3', reverse 5'-GTAGGTGAACCTGCAGAAGGATCA-3'. The PCR products were purified on agarose gels followed by phenol/chloroform extraction. For the Northern blot analysis the PCR products were labelled by $[\alpha-$ $\left.{ }^{32} \mathrm{P}\right] \mathrm{dATP}$ using the Random Primed DNA Labelling Kit (Boehringer). In a nuclear run-on assay, PCR products were inserted into a pGEM-T vector (TA cloning kit; Stratagene) and immobilized on nitrocellulose as a probe. Nucleotide sequences of all PCR products were verified by sequencing.

Isolation of RNA and Northern blot analysis. Total RNA was isolated by a modified guanidium thiocyanate procedure (Wang \& Wang, 1985b). Total RNA $(0.5 \mu \mathrm{g})$ was sizefractionated on a $1.2 \%$ agarose $/ 2 \cdot 2 \mathrm{M}$ formaldehyde gel and transferred to a nylon membrane (Hybond-N; Amersham). Blots were hybridized with radiolabelled DNA probes for ME and PFOR and subsequently washed as described by Johnson et al. (1990). Subsequently, the membranes were stripped down and rehybridized with DNA probe for $\beta$-tubulin as a control.

Nuclear run-on assay. Synthesis of nascent mRNA was investigated by the permeable cells technique (Vaňácová et al., 2001a). Briefly, trichomonads were permeabilized with lysolecithin and incubated in presence of $\left[\alpha^{32}\right.$ P]UTP. Nascent RNA was isolated by TRIzol extraction (Gibco-BRL) and ${ }^{32} \mathrm{P}-$ labelled transcripts were hybridized to DNA-specific probes immobilized on nitrocellulose. To assure standard conditions DNA concentrations of all probes were determined spectro- photometrically and an equal amount of each probe was applied onto nitrocellulose using a Bio-Rad filtration apparatus. Probes for $16 \mathrm{~S}$ rRNA and tubulin were used as positive controls; the probe for the pGEM vector, employed for subcloning of all probes, served as a negative control. Blots were analysed using storage phosphor autoradiography (PhosphorImager SI; Molecular Dynamics).

\section{RESULTS}

\section{Development of resistance}

Trichomonads exposed to metronidazole developed resistance gradually, passing through a sequence of stages marked by progressively increasing resistance and characteristic changes in their metabolic properties. Increasing drug resistance of the representative strains (Table 1) derived in vitro from the drug-susceptible Trichomonas vaginalis strain TV 10-02 is shown in Table 2. We found that the aerobic type of resistance developed as a part of a common process, appearing in advance of anaerobic resistance. Relatively short maintenance of trichomonads at low concentrations of metronidazole ( 50 days at $3 \mu \mathrm{g} \mathrm{ml}^{-1}$ ) induced resistance with an aerobic MLC of over $200 \mu \mathrm{g}$ metronidazole $\mathrm{ml}^{-1}$ $(\sim 1.2 \mathrm{mM})$, while the anaerobic MLC remained low $\left(\sim 4 \mu \mathrm{g}\right.$ metronidazole $\mathrm{ml}^{-1}$ ). Development of anaerobic resistance required further exposure to a stepped increase in pressure of the drug. The strains with advanced anaerobic resistance (MR-30, MR-50, MR-100) showed similar MLCs (1120-1425 $\mu \mathrm{g}$ metronidazole $\mathrm{ml}^{-1}$, i.e. $6.5-8.3 \mathrm{mM}$ ), but differed in their ability to reproduce in the presence of the drug under anaerobiosis (strains MR-30, MR-50 and MR-100 grew in TYM medium at 30,50 and $100 \mu \mathrm{g}$ metronidazole $\mathrm{ml}^{-1}$, respectively). The ability to grow at $100 \mu \mathrm{g}$ metronidazole $\mathrm{ml}^{-1}$ (strain MR-100) was acquired after exposure to the drug for 359 days, but further passages at this concentration of the drug were necessary to obtain fully developed resistance, stable in the absence of drug pressure. The strains displaying anaerobic resistance also showed increased aerobic MLCs. In parallel with the increasing resistance, their sensitivity to oxygen increased so that the assays for aerobic MLCs had to be run in an atmosphere with lowered $\mathrm{O}_{2}$ content (Table 2).

\section{Hydrogenosomal enzyme activities}

Activities of the hydrogenosomal enzymes PFOR, hydrogenase, NADH:FOR and $\mathrm{NAD}^{+}$-dependent ME in the parent strain TV 10-02 and its resistant derivatives are given in Table 3.

The aerobically resistant derivative MR-3 showed decreased activities of all enzymes monitored except for hydrogenase. The maximum decrease in specific activity (to about $20 \%$ of that found in the parent strain) was seen for PFOR. Activities of NADH:FOR and ME decreased to 56 and $30 \%$ of parent activity, respectively.

In the MR-5 strain, representing the early stage of anaerobic resistance, no activity of PFOR was detected and the activity of hydrogenase decreased profoundly (14\% of that found in the parent strain). Activities of 
Table 2. MLC of metronidazole for the drug-susceptible Trichomonas vaginalis strain TV 10-02 and its in vitro-developed resistant derivatives

The assay was performed in 95-well round bottom microtitre plates under anaerobic (anaerobic MLC) or aerobic conditions (aerobic MLC) as described in Methods. The MLC was defined as the lowest dilution at which no motile cells were observed. $n$, Number of independent experiments.

\begin{tabular}{|c|c|c|c|c|c|}
\hline \multirow[t]{3}{*}{ Strain } & & \multicolumn{4}{|c|}{ MLC at $48 \mathrm{~h}\left(\mu \mathrm{g} \mathrm{ml}^{-1}\right)$} \\
\hline & & \multicolumn{2}{|c|}{ Anaerobic } & \multicolumn{2}{|c|}{ Aerobic } \\
\hline & & $\begin{array}{l}\text { Geometric } \\
\text { mean }(n)\end{array}$ & Range & $\begin{array}{c}\text { Geometric } \\
\text { mean }(n)\end{array}$ & Range \\
\hline Sensitive & TV 10-02 & $1 \cdot 7(3)$ & $0 \cdot 78-6 \cdot 25$ & $4 \cdot 7^{*}(3)$ & $1 \cdot 56-6 \cdot 25$ \\
\hline \multirow[t]{5}{*}{ Resistant } & MR-3 & $4 \cdot 2(3)$ & $3 \cdot 12-6 \cdot 25$ & $261 \cdot 5 *(3)$ & $200-400$ \\
\hline & MR-5 & $15 \cdot 7(3)$ & $6 \cdot 25-25$ & $96 \cdot 2+(3)$ & $50-200$ \\
\hline & MR-30 & $1120 \cdot 4(2)$ & $400-1600$ & $321 \cdot 2+(2)$ & $200-400$ \\
\hline & MR-50 & $1400 \cdot 1(3)$ & $800-1600$ & $405 \cdot 7 \dagger(3)$ & $200-800$ \\
\hline & MR-100 & $1425 \cdot 4(3)$ & $800-1600$ & $416 \cdot 0+(3)$ & $200-800$ \\
\hline
\end{tabular}

*Exposed to air.

† Exposed to $5 \% \mathrm{O}_{2}$ in $\mathrm{N}_{2}$ with $5 \% \mathrm{CO}_{2}$.

Table 3. Hydrogenosomal enzyme activities of the metronidazole-susceptible Trichomonas vaginalis strain TV 10-02 and its drug-resistant derivatives

Data are presented $\pm S D ; n$, number of independent experiments; ND, no detectable activity (limits of detection, $0.5 \mathrm{nmol})$.

\begin{tabular}{|c|c|c|c|c|}
\hline \multirow[t]{2}{*}{ Strain } & \multicolumn{4}{|c|}{ Enzyme activity $\left[\mathrm{nmol} \mathrm{min}^{-1}(\mathrm{mg} \text { protein })^{-1}\right](n)$} \\
\hline & PFOR & Hydrogenase & NADH:FOR & $\mathrm{NAD}^{+}$-dependent $\mathrm{ME}$ \\
\hline TV 10-02 & $527 \pm 243(5)$ & $1438 \pm 29(5)$ & $603 \pm 29(3)$ & $1314 \pm 399(5)$ \\
\hline MR-3 & $107 \pm 14(4)$ & $1461 \pm 18(2)$ & $342 \pm 167(3)$ & $396 \pm 126(3)$ \\
\hline MR-5 & ND (4) & $202 \pm 6(3)$ & $251 \pm 73 \cdot 1(3)$ & $279 \pm 76(3)$ \\
\hline MR-30 & ND $(2)$ & $125 \pm 49(6)$ & $295 \pm 114(5)$ & $21 \pm 8(4)$ \\
\hline MR-50 & $\mathrm{ND}(2)$ & ND (3) & $156 \pm 34(2)$ & $34 \pm 11(4)$ \\
\hline MR-100 & $\mathrm{ND}(4)$ & ND (3) & ND (3) & ND (3) \\
\hline
\end{tabular}

ME and NADH:FOR decreased only slightly when compared with the aerobic stage (MR-3). The activity of hydrogenase continued to decline with increasing anaerobic resistance and was completely lost at the stage represented by the MR-50 strain. A marked decrease of $\mathrm{ME}$ activity was determined in the MR-30 strain (less than $2 \%$ of parent activity), while NADH:FOR activity still amounted to about $26 \%$ of that of the parent in the MR-50 strain. Both activities were undetectable in the MR-100 strain with fully developed anaerobic resistance (limits of detection, $0.5 \mathrm{nmol}$ ).

\section{Cytoplasmic enzyme activities}

The activities of three cytoplasmic enzymes, LDH, pyruvate kinase and $\mathrm{NADP}^{+}$-dependent $\mathrm{ME}$, determined in the drug-susceptible strain TV 10-02 and its derivatives at different levels of resistance development, are listed in Table 4. The data show a progressive increase in LDH activity during development of anaerobic resistance, up to values exceeding those in the parent drug-susceptible strains sevenfold. The activities of pyruvate kinase increased in the resistant strains about threefold on average. The resistant strains also showed slightly increased activities of the cytosolic ME. Both activities showed minor fluctuations between the individual drug-resistant strains with a peak at the stage of early anaerobic resistance (MR-5).

\section{Metabolic end products}

The release of the main end products of Trichomonas vaginalis carbohydrate metabolism by the drug-susceptible strain and its resistant derivatives is compared 
Table 4. Cytoplasmic enzyme activities of the metronidazole-susceptible Trichomonas vaginalis strain TV 10-02 and its drug-resistant derivatives

Data are presented $\pm \mathrm{SD} ; n$, number of independent experiments.

\begin{tabular}{|c|c|c|c|}
\hline \multirow[t]{2}{*}{ Strain } & \multicolumn{3}{|c|}{ Enzyme activity $\left[\mathrm{nmol} \mathrm{min}^{-1}(\mathrm{mg} \text { protein })^{-1}\right](n)$} \\
\hline & $\mathrm{LDH}$ & Pyruvate kinase & NADP-dependent ME \\
\hline TV 10-02 & $792 \pm 234(5)$ & $165 \pm 25(6)$ & $491 \pm 61(3)$ \\
\hline MR-3 & $680 \pm 154(4)$ & $527 \pm 38(3)$ & $692 \pm 53(2)$ \\
\hline MR-5 & $1064 \pm 46(4)$ & $666 \pm 131(5)$ & $817 \pm 40(3)$ \\
\hline MR-30 & $2983 \pm 268(3)$ & $478 \pm 92(5)$ & $532 \pm 94(5)$ \\
\hline MR-50 & $4264 \pm 318(3)$ & $582 \pm 63(4)$ & $574 \pm 73(2)$ \\
\hline MR-100 & $5663 \pm 347(3)$ & $411 \pm 5(3)$ & $674 \pm 85(3)$ \\
\hline
\end{tabular}

Table 5. Metabolic end products of the metronidazole-susceptible Trichomonas vaginalis strain TV 10-02 and its drug-resistant derivatives

Data are presented $\pm \mathrm{SD} ; n$, number of independent experiments.

\begin{tabular}{|c|c|c|c|c|}
\hline \multirow[t]{2}{*}{ Strain } & \multicolumn{4}{|c|}{ End product $\left[\mathrm{nmol} \mathrm{min}^{-1}(\mathrm{mg} \text { protein })^{-1}\right](n)$} \\
\hline & Lactate & Glycerol & Acetate & Hydrogen" \\
\hline TV 10-02 & $40 \cdot 26 \pm 9 \cdot 93(21)$ & $5 \cdot 27 \pm 0 \cdot 91(15)$ & $5 \cdot 79 \pm 0 \cdot 91(20)$ & $1.83 \pm 0.58(10)$ \\
\hline MR-3 & $59 \cdot 92 \pm 18 \cdot 54(8)$ & $7 \cdot 11 \pm 1 \cdot 77(8)$ & $7 \cdot 56 \pm 1 \cdot 97(8)$ & $1 \cdot 17 \pm 0 \cdot 32(4)$ \\
\hline MR-5 & $87 \cdot 53 \pm 22 \cdot 91(8)$ & $5 \cdot 09 \pm 2 \cdot 06(8)$ & $4 \cdot 30 \pm 1 \cdot 05(8)$ & $0 \cdot 67 \pm 0.04(4)$ \\
\hline MR-30 & $105 \cdot 24 \pm 26 \cdot 37(8)$ & $5 \cdot 06 \pm 0.95(7)$ & $0 \cdot 59 \pm 0 \cdot 19(8)$ & Undetectable (8) \\
\hline MR-50 & $112 \cdot 31 \pm 10 \cdot 77(4)$ & $6 \cdot 25 \pm 1 \cdot 67(5)$ & $0 \cdot 66 \pm 0 \cdot 11(4)$ & Undetectable (6) \\
\hline MR-100 & $115 \cdot 32 \pm 0 \cdot 26(4)$ & $3.57 \pm 0.57(3)$ & $0 \cdot 15 \pm 0 \cdot 04(7)$ & Undetectable (5) \\
\hline
\end{tabular}

*Detection limits for hydrogen were approximately $50 \mathrm{nmol}$. The numbers given in the table were recalculated from values obtained by $60 \mathrm{~min}$ incubation of the cell suspension corresponding to $\sim 10 \mathrm{mg}$ protein.

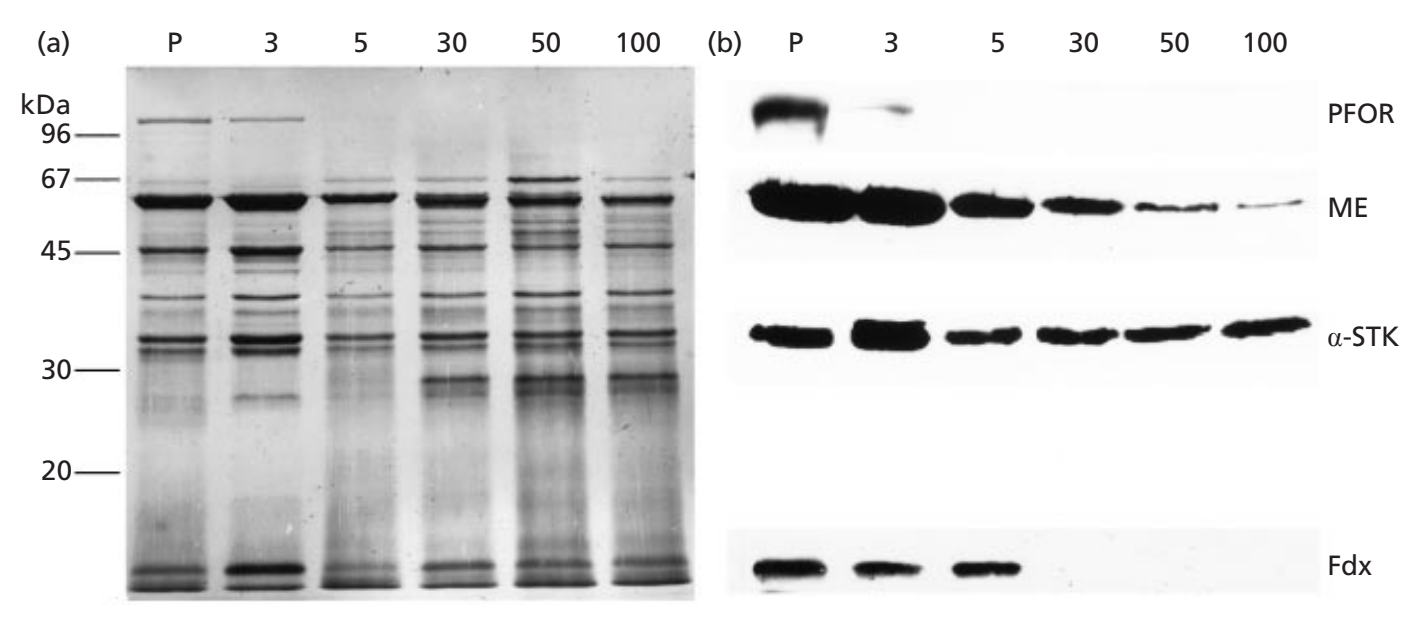

Fig. 1. SDS-PAGE (a, silver-stained gel) and Western blot (b) analysis of the purified hydrogenosomal fractions isolated from the metronidazole-susceptible Trichomonas vaginalis strain TV 10-02 and its drug-resistant derivatives MR-3, MR-5, MR-30, MR-50 and MR-100 displaying the aerobic (3), early anaerobic (5), advanced anaerobic (30, 50) and fully developed anaerobic resistance (100) to metronidazole. About $10 \mu \mathrm{g}$ protein was loaded per lane. $\alpha$-STK, $\alpha$-subunit of STK; Fdx, ferredoxin. 
in Table 5. The results showed an increase in lactate production with increasing resistance while the production of hydrogenosomal metabolites, hydrogen and acetate, decreased or eventually ceased. Hydrogen production stopped when trichomonads reached the stage of moderately advanced anaerobic resistance (MR30). At the same resistance level acetate production dropped dramatically. Release of this end product decreased further up to the limit of detectability in the strain with fully developed anaerobic resistance (MR100). No substantial changes in the production of glycerol were found between the drug-susceptible and -resistant strains.

\section{Protein profiles of hydrogenosomes}

To examine whether the decrease or loss of activities of some hydrogenosomal enzymes observed during the development of resistance is a direct result of lowered levels of particular proteins, we analysed extracts of purified hydrogenosomes from the parent and resistant trichomonads by Western blotting. These experiments were also aimed at determination of whether there are any resistance-related changes in the expression of ferredoxin, the electron carrier involved in metronidazole activation. The blots were probed with antibodies against PFOR, ME and ferredoxin. The probe for STK, the hydrogenosomal enzyme uncommitted in metronidazole activation, was included as a control (Fig. 1). The $\mathrm{mAb}$ against PFOR identified a $120 \mathrm{kDa}$ band present in the parent (TV 10-02) and the aerobically resistant (MR-3) strains. In accord with the absence of enzyme activities, the band was missing in all subsequent stages of resistance development, including that of early anaerobic resistance (MR-5). The antibody against ME detected a $61 \mathrm{kDa}$ band. It was of the same intensity in the parent and the aerobically resistant strain (MR-3), but decreased progressively with increasing anaerobic resistance. The ferredoxin antibody revealed a $12 \mathrm{kDa}$ band of similar intensity present in the parent and in resistant strains with aerobic (MR-3) and early anaerobic (MR-5) resistance, but missing in strains representing more advanced stages of anaerobic resistance (MR-30, MR-50, MR-100). A $32.5 \mathrm{kDa}$ polypeptide visualized by the antibody against the $\alpha$-subunit of STK was present almost uniformly in the hydrogenosomes of the parent and the resistant strains, regardless of their resistance levels.

\section{Activation of metronidazole}

To determine whether the hydrogenosomal deficiencies detected in the resistant strains interfere with metronidazole reduction, we monitored the release of metronidazole free radicals by EPR spectroscopy after incubation of susceptible or resistant trichomonads with the drug. The organisms were incubated in a glucosecontaining buffer under anaerobic conditions. Characteristic signals of metronidazole free radicals produced by the drug-susceptible parent strain (TV 10-02) are

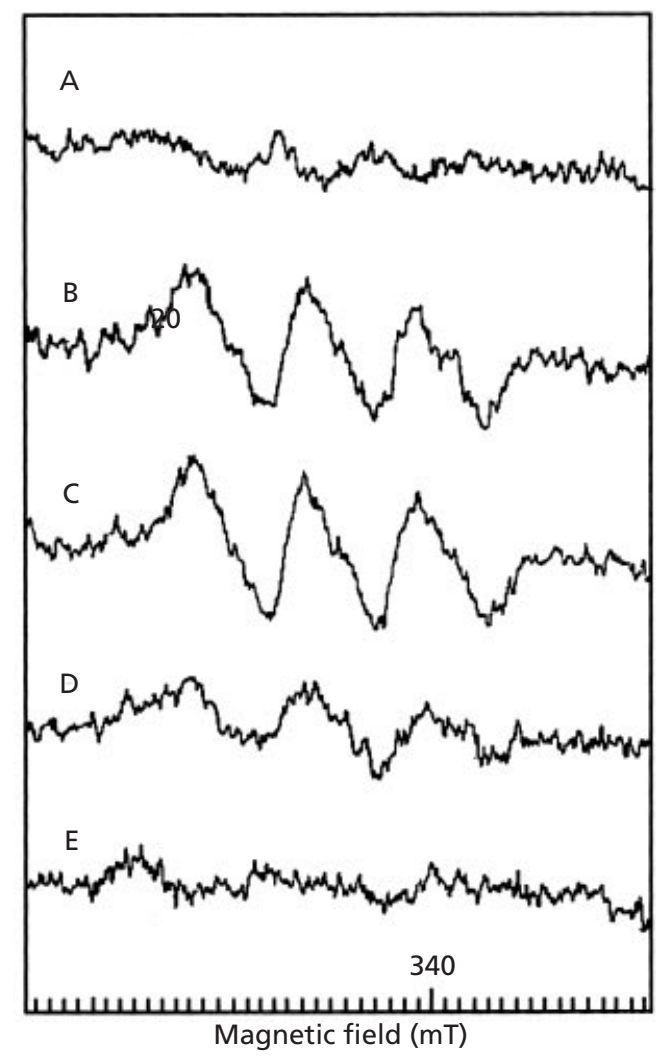

Fig. 2. EPR spectra demonstrating generation of metronidazole anion radicals by drug-susceptible Trichomonas vaginalis strain TV 10-02 and its derivatives at different levels of resistance. A, TV 10-02 control cells without addition of metronidazole; B, TV 10-02 cells with $12 \mathrm{mM}$ metronidazole; C, MR-3 aerobic resistance (12 mM metronidazole); D, MR-5 early anaerobic resistance (12 mM metronidazole); E, MR-100 fully developed anaerobic resistance (12 $\mathrm{mM}$ metronidazole). Instrument settings and conditions were as follows: field set, $3390 \mathrm{G}$; scan scale, $100 \mathrm{G}$; modulation amplitude, $4 \mathrm{G}$; microwave power, $6.324 \mathrm{~mW}$; microwave frequency, $9.52 \mathrm{Ghz}$; receiver gain, $8 \times 10^{3}$; scan time, $2 \mathrm{~min}$; time constant, $1 \mathrm{~s} ; 6 \times 10^{7}$ cells per cuvette.

shown in Fig. 2(B). No signal was observed in the absence of metronidazole (Fig. 2A). The signal produced by the aerobically resistant derivative (MR-3) was almost identical to that of the parent strain (Fig. 2C). Surprisingly, the signal was also produced by the MR-5 strain (early anaerobic resistance) deficient in PFOR. It was weaker in comparison with that of the parent strain, but still distinct. No signal of metronidazole radicals was detected with the MR-100 strain with fully developed anaerobic resistance (Fig. 2D).

\section{Nucleic acids analysis}

To define further mechanisms involved in the acquisition of metronidazole resistance, we analysed the expression of genes encoding PFOR and hydrogenosomal ME, the two hydrogenosomal oxidoreductases down-regulated 
(a)

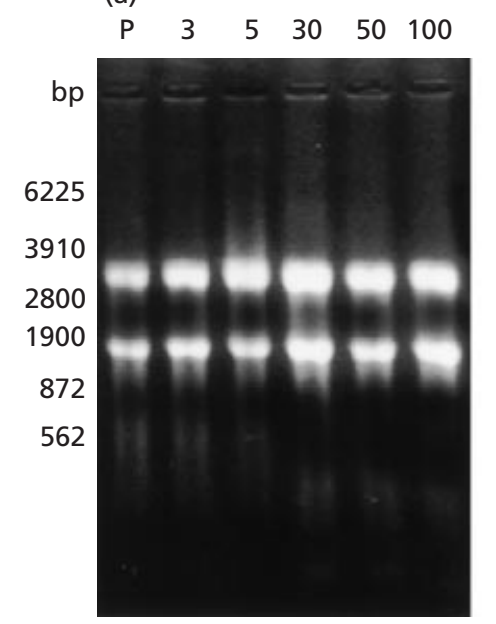

(b)

$\begin{array}{llllll}P & 3 & 5 & 30 & 50 & 100\end{array}$

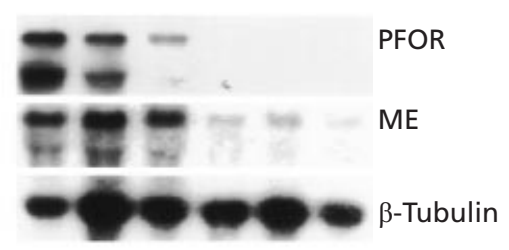

Fig. 3. (a) Agarose gel fractionation of total RNA isolated from parental Trichomonas vaginalis strain TV 10-02 (P) and five resistant derivatives with increasing resistance to metronidazole (MR-3, MR-5, MR-30, MR50 and MR-100). (b) Comparison of steadystate concentrations of mRNA encoding PFOR and hydrogenosomal ME in the same set of Trichomonas vaginalis strains. Amounts of total RNA loaded onto each line were standardized according to the level of $\beta$ tubulin mRNA.

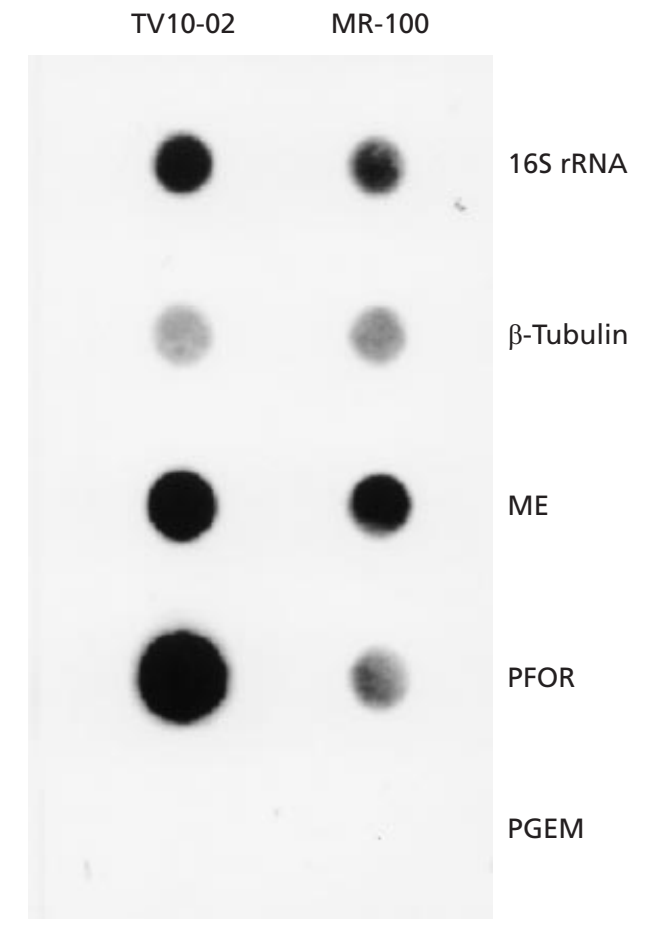

Fig. 4. Transcription of $M E$ and $P F O R$ genes in Trichomonas vaginalis strain TV 10-02 sensitive to metronidazole and its resistant derivative with fully developed anaerobic resistance, MR-100. The synthesis of nascent mRNA was compared by nuclear run-on assay in lysolecithin-permeabilized cells. Dot blots contained DNA clones with fragments of $16 \mathrm{~S}$ rRNA, $\beta$ tubulin, ME and PFOR genes. Blots were hybridized with nascent ${ }^{32}$ P-labelled transcripts. Plasmid pGEM DNA was used as negative control.

in the process of resistance development. The Northern blot analysis (Fig. 3) revealed a marked decrease in the steady-state level of ME mRNA in strains MR-30 and MR-50, representing more advanced stages of anaerobic resistance, while the hybridization signal of PFOR mRNA was already abolished at the early stage of anaerobic resistance (strain MR-5). The amounts of control mRNA for $\beta$-tubulin remained unchanged. Investigations of nascent mRNA synthesis in the drugsusceptible strain and its fully resistant derivative (MR100) revealed significantly lowered transcription of genes encoding PFOR. No apparent change in the transcription was observed in genes encoding $\mathrm{ME}$ and the two positive controls, SSU rRNA and $\beta$-tubulin (Fig. 4).

\section{DISCUSSION}

The results presented in this paper provide evidence that trichomonads develop resistance to metronidazole in a multistep process based on gradual decrease and eventual loss of activities of the hydrogenosomal proteins involved in drug-activating pathways. Consequently, the release and transport of electrons required for drug reduction is inhibited and microbicidal radicals cannot be generated. Monitoring of resistance development in Trichomonas vaginalis from a drug-sensitive parent strain (TV 10-02) up to the final stage of fully developed anaerobic resistance (MR-100) revealed two unexpected phenomena. (1) Aerobic and anaerobic resistance, considered to be unrelated, developed in a common continuous process. (2) The lack of PFOR activity, the major donor of electrons for drug reduction, did not result in fully developed anaerobic resistance.

Aerobic resistance appeared as the earliest stage of resistance development (MR-3). The properties of in vitro-developed strains are similar to those of clinical isolates obtained from treatment of refractory patients. Both contain PFOR activity and are susceptible to the drug under anaerobic conditions. Their resistance is detectable in mice, but in vitro only if some oxygen is present (Tachezy et al., 1993). The strain developed in this study (MR-3) showed considerably lower activities of hydrogenosomal enzymes, except hydrogenase, in comparison with those of the parent strain. However, the pathway activating metronidazole was functional as shown by the presence of nitro free radical signals in EPR spectra and by susceptibility of the organisms to 
metronidazole under anaerobic conditions. The ferredoxin of this strain was expressed at a similar level as in the parent strain (Fig. 1). Moreover, $\mathrm{Fe}-\mathrm{S}$ clusters of this strain and of examined clinical isolates displaying aerobic resistance were intact, as shown by characteristic signals in EPR spectra (Rasoloson et al., 2001). Thus, the reports pointing to a low specific activity of hydrogenase (Ellis et al., 1992) or ferredoxin insufficiency (Quon et al., 1992; Yarlett et al., 1986b) in some aerobically resistant isolates rather reflect individual strain variability than specific attributes of aerobic resistance.

The following stage, early anaerobic resistance (MR-5), is characterized by lack of PFOR activity. Surprisingly the drug resistance of these organisms remained relatively low (MLC $15 \cdot 7 \mu \mathrm{g} \mathrm{ml}^{-1}$ ). The metronidazole radical signal, albeit of a lower intensity, was still apparent in EPR spectra if the trichomonads were exposed to metronidazole. The ability of PFOR-deficient trichomonads to activate metronidazole indicates involvement of an alternative pathway, providing electrons for reduction of the drug. Our results strongly suggest that the hydrogenosomal $\mathrm{NAD}^{+}$-dependent $\mathrm{ME}$, catalysing oxidative decarboxylation of malate to pyruvate, performs this function. By the activity of $\mathrm{NADH}$ :FOR, reoxidizing NADH produced in this reaction, reduced equivalents can be transferred to ferredoxin to be available for drug activation. As is evident from data presented in this paper, trichomonads at the early stage of anaerobic resistance (MR-5) possess all components of this pathway. Gradual elimination of these proteins during further development of the anaerobic resistance stresses their causal relationship with metronidazole activation at the early PFOR-deficient stage (MR-5). Consequently, acquisition of fully developed anaerobic resistance in Trichomonas vaginalis (MR-100) requires elimination of both ferredoxin-linked electron-generating systems in hydrogenosomes.

The decrease or loss of hydrogenosomal metabolism in metronidazole-resistant strains is compensated by an increased rate of glycolysis (Kulda et al., 1989) and by enhancement of a cytoplasmic pathway metabolizing pyruvate (Kulda et al., 1989; Kulda, 1999). Metronidazole-resistant strains of both Trichomonas vaginalis and Tritrichomonas foetus tune their catabolic process to production of a single dominant end product. Our metabolic data showed a four- to fivefold increase in the activity of pyruvate kinase and confirmed marked enhancement of cytosolic lactate fermentation in the resistant strains. The elevated lactate production was underlined by a progressive increase in LDH activity. While lactate is the main glycolytic end product of metronidazole-resistant Trichomonas vaginalis, resistant Tritrichomonas foetus enhances fermentation to ethanol (Čerkasovová et al., 1984). A metabolic switch to cytoplasmic pyruvate metabolism is apparently a more general strategy used by trichomonads confronted with hydrogenosomal insufficiency. We have observed a similar phenomenon in Tritrichomonas foetus exposed to iron-restricted conditions (Vaňáčová et al., 2001b).
Some metabolic end products of trichomonads developing anaerobic resistance do not seem to be consistent with the lack of PFOR activity, but alternative metabolic processes can explain their production. Hydrogen production at the early stage of anaerobic resistance (MR5 ) is apparently due to the activity of the ME pathway. It is likely that in the absence of the drug, electrons are transported by ferredoxin to hydrogenase that is still active at this stage. Accordingly, production of hydrogen is stopped at the more advanced stage (MR-30) deficient in ferredoxin. Production of acetate detected at early (MR-5) up to more advanced stages of resistance development (MR-30, MR-50) can be accounted for by the activity of alternative 2-ketoacid oxidoreductases reported by Brown et al. (1999) that are active in metronidazole-resistant Trichomonas vaginalis and do not require ferredoxin as electron acceptor. However, the fate of these activities during development of resistance has not been followed in this study.

To examine at which level down-regulation of major hydrogenosomal activities associated with drug resistance occurs, we followed changes in protein and mRNA levels by Western and Northern blotting and examined transcription of genes for PFOR and hydrogenosomal ME by nuclear run-on assay. We found that the decrease or loss of enzyme activities is due to decreased expression of pertinent proteins. We also observed loss of ferredoxin at more advanced stages of anaerobic resistance (MR-30 and higher). In contrast, the protein levels of STK, the hydrogenosomal enzyme uncommitted in metronidazole activation, remained unaffected. In parallel to the decreased protein levels, steady-state levels of PFOR and ME mRNAs decreased with increasing resistance to the drug. The nuclear run-on assays monitoring synthesis of nascent mRNA revealed that the loss of PFOR results from down-regulation of gene transcription. In contrast, transcription of the genes encoding ME was not markedly changed. Thus the expression of ME appears to be regulated at different levels, most probably at the level of mRNA stability. Whilst this manuscript was in preparation for publication, Land et al. (2001) reported decreased transcription of PFOR and loss of other hydrogenosomal proteins associated with metronidazole activation in a drug-resistant Tritrichomonas foetus. Down-regulation of PFOR and ferredoxin in strains induced for anaerobic resistance has also been observed in another Trichomonas vaginalis strain (Brown et al., 1999) and in Giardia (Liu et al., 2000), and apparently it is a common feature of high-level resistance to the drug. As shown in this paper, acquisition of anaerobic resistance in Trichomonas vaginalis involves in addition down-regulation of the hydrogenosomal ME.

Gradual build up of metronidazole resistance through the sequence of stages characterized here suggests that metronidazole resistance is based on stepwise accumulation of mutations that affect expression of genes for hydrogenosomal proteins involved in drug activation. Up-regulation of cytosolic pathways of pyruvate metabolism that accompany the development of resistance 
may be attributed to metabolic feedback mechanisms as has been observed in hydrogenosomal deficiencies of different origin (Vaňáčová et al., 2001b). Analysis of regulatory sequences (Liston et al., 1999; Liston \& Johnson, 1999; Quon et al., 1994) of genes for proteins involved in metronidazole resistance may bring further insight into the molecular mechanisms of this process. Work along these lines is in progress.

\section{ACKNOWLEDGEMENTS}

This work was supported by the National Grant Agency of the Czech Republic (204/97/0263) and in part by grants vs 96142 and OC B9.10 provided by the Ministry of Education of the Czech Republic. The EPR spectroscopy was done in the laboratory of Professor Richard Cammack, King's College London, whose expert guidance is gratefully acknowledged. Thanks are also due to Professor Ladislav Feltl (Department of Analytical Chemistry, Charles University in Prague) for generous help with the determination of hydrogen production by gas chromatography.

\section{REFERENCES}

Bergmeyer, H. U. (1963). Methods of Enzymatic Analysis. New York: Academic Press.

Bowtell, D. D. (1987). Rapid isolation of eukaryotic DNA. Anal Biochem 162, 463-465.

Brown, D. M., Upcroft, J. A., Dodd, H. N., Chen, N. \& Upcroft, P. (1999). Alternative 2-keto acid oxidoreductase activities in Trichomonas vaginalis. Mol Biochem Parasitol 98, 203-214.

Čerkasov, J., Čerkasovová, A., Kulda, J. \& Vilhelmová, D. (1978). Respiration of hydrogenosomes of Tritrichomonas foetus. J Biol Chem 253, 1207-1214.

Čerkasovová, A., Čerkasov, J. \& Kulda, J. (1984). Metabolic differences between metronidazole resistant and susceptible strains of Tritrichomonas foetus. Mol Biochem Parasitol 11, 105-118.

Čerkasovová, A., Novák, J., Čerkasov, J., Kulda, J. \& Tachezy, J. (1987). Metabolic properties of Trichomonas vaginalis resistant to metronidazole under anaerobic conditions. Acta Univ Carol Biol 30, 505-512.

Chapman, A., Cammack, R., Linstead, D. \& Lloyd, D. (1985). The generation of metronidazole radicals in hydrogenosomes isolated from Trichomonas vaginalis. J Gen Microbiol 131, 2141-2144.

de Carneri, I., Achilli, G., Monti, G. \& Trane, F. (1969). Induction of in-vivo resistance of Trichomonas vaginalis to metronidazole. Lancet 2, 1308-1309.

Diamond, L. S. (1957). The establishment of various trichomonads of animals and man in axenic cultures. J Parasitol 43, 488-490.

Doran, D. J. (1959). Studies on trichomonads: III. inhibitors, acid production, and substrate utilization by 4 strains of Tritrichomonas foetus. J Protozool 6, 177-182.

Drmota, T., Proost, P., Van Ranst, M., Weyda, F., Kulda, J. \& Tachezy, J. (1996). Iron-ascorbate cleavable malic enzyme from hydrogenosomes of Trichomonas vaginalis: purification and characterization. Mol Biochem Parasitol 83, 221-234.

Edwards, D. I. (1993). Nitroimidazole drugs - action and resistance mechanisms. II. Mechanisms of resistance. J Antimicrob Chemother 31, 201-210.

Ellis, J. E., Cole, D. \& Lloyd, D. (1992). Influence of oxygen on the fermentative metabolism of metronidazole-sensitive and resistant strains of Trichomonas vaginalis. Mol Biochem Parasitol 56, 79-88.

Johnson, P. J., d'Oliveira, C. E., Gorrell, T. E. \& Müller, M. (1990). Molecular analysis of the hydrogenosomal ferredoxin of the anaerobic protist Trichomonas vaginalis. Proc Natl Acad Sci U S A 87, 6097-6101.

Kabíčková, H., Kulda, J., Čerkasovová, A. \& Nẽmcová, H. (1987). Metronidazole resistant Tritrichomonas foetus: activities of hydrogenosomal enzymes in course of development of anaerobic resistance. Acta Univ Carol Biol 30, 513-519.

Kulda, J. (1999). Trichomonads, hydrogenosomes and drug resistance. Int J Parasitol 29, 199-212.

Kulda, J., Vojtěchovská, M., Tachezy, J., Demeš, P. \& Kunzová, E. (1982). Metronidazole resistance of Trichomonas vaginalis as a cause of treatment failure in trichomoniasis. A case report. $\mathrm{Br} \mathrm{J}$ Vener Dis 58, 394-399.

Kulda, J., Čerkasov, J., Demeš, P. \& Čerkasovová, A. (1984). Tritrichomonas foetus: stable anaerobic resistance to metronidazole in vitro. Exp Parasitol 57, 93-103.

Kulda, J., Kabíčková, H., Tachezy, J., Čerkasovová, A. \& Čerkasov, J. (1989). Metronidazole resistant trichomonads: mechanisms of in vitro developed anaerobic resistance. In Biochemistry and Molecular Biology of 'Anaerobic' Protozoa, pp. 137-160. Edited by D. Lloyd, G. H. Coombs \& T. A. P. Paget. Chur, Switzerland: Harwood Academic Publishers.

Kulda, J., Tachezy, J. \& Čerkasovová, A. (1993). In vitro induced anaerobic resistance to metronidazole in Trichomonas vaginalis. J Eukaryot Microbiol 40, 262-269.

Lahti, C. J., d'Oliveira, C. E. \& Johnson, P. J. (1992). Beta-succinylcoenzyme A synthetase from Trichomonas vaginalis is a soluble hydrogenosomal protein with an amino-terminal sequence that resembles mitochondrial presequences. J Bacteriol 174, 68226830.

Land, K. M., Clemens, D. L. \& Johnson, P. J. (2001). Loss of multiple hydrogenosomal proteins associated with organelle metabolism and high-level drug resistance in trichomonads. Exp Parasitol 97, 102-110.

Lindmark, D. G. \& Müller, M. (1973). Hydrogenosome, a cytoplasmic organelle of the anaerobic flagellate Tritrichomonas foetus, and its role in pyruvate metabolism. J Biol Chem 248, 7724-7728.

Liston, D. R. \& Johnson, P. J. (1999). Analysis of a ubiquitous promoter element in a primitive eukaryote: early evolution of the initiator element. Mol Cell Biol 19, 2380-2388.

Liston, D. R., Carrero, J. C. \& Johnson, P. J. (1999). Upstream regulatory sequences required for expression of the Trichomonas vaginalis alpha-succinyl CoA synthetase gene. Mol Biochem Parasitol 104, 323-329.

Liu, S. M., Brown, D. M., O’Donoghue, P., Upcroft, P. \& Upcroft, J. A. (2000). Ferredoxin involvement in metronidazole resistance of Giardia duodenalis. Mol Biochem Parasitol 108, 137-140.

Lloyd, D. \& Kristensen, B. (1985). Metronidazole inhibition of hydrogen production in vivo in drug-sensitive and -resistant strains of Trichomonas vaginalis. J Gen Microbiol 131, 849-853.

Lossick, J. G., Müller, M. \& Gorrell, T. E. (1986). In vitro drug susceptibility and doses of metronidazole required for cure in cases of refractory vaginal trichomoniasis. J Infect Dis 153, 948-955.

Meingassner, J. G. \& Thurner, J. (1979). Strain of Trichomonas vaginalis resistant to metronidazole and other 5 -nitroimidazoles. Antimicrob Agents Chemother 15, 254-257.

Meingassner, J. G., Mieth, H., Czok, R., Lindmark, D. G. \& Müller, 
M. (1978). Assay conditions and the demonstration of nitroimidazole resistance in Tritrichomonas foetus. Antimicrob Agents Chemother 13, 1-3.

Meri, T., Jokiranta, T. S., Suhonen, L. \& Meri, S. (2000). Resistance of Trichomonas vaginalis to metronidazole: report of the first three cases from Finland and optimization of in vitro susceptibility testing under various oxygen concentrations. J Clin Microbiol 38, 763-767.

Mertens, E., Van Schaftingen, E. \& Müller, M. (1992). Pyruvate kinase from Trichomonas vaginalis, an allosteric enzyme stimulated by ribose 5-phosphate and glycerate 3-phosphate. Mol Biochem Parasitol 54, 13-20.

Moreno, S. N., Mason, R. P., Muniz, R. P., Cruz, F. S. \& Docampo, R. (1983). Generation of free radicals from metronidazole and other nitroimidazoles by Tritrichomonas foetus. J Biol Chem 258, 4051-4054.

Müller, M. (1986). Reductive activation of nitroimidazoles in anaerobic microorganisms. Biochem Pharmacol 35, 37-41.

Müller, M. (1993). The hydrogenosome. J Gen Microbiol 139, 2879-2889.

Müller, M., Meingassner, J. G., Miller, W. A. \& Ledger, W. J. (1980). Three metronidazole-resistant strains of Trichomonas vaginalis from the United States. Am J Obstet Gynecol 138, 808-812.

Quon, D. V., d'Oliveira, C. E. \& Johnson, P. J. (1992). Reduced transcription of the ferredoxin gene in metronidazole-resistant Trichomonas vaginalis. Proc Natl Acad Sci U S A 89, 4402-4406.

Quon, D. V., Delgadillo, M. G., Khachi, A., Smale, S. T. \& Johnson, P. J. (1994). Similarity between a ubiquitous promoter element in an ancient eukaryote and mammalian initiator elements. Proc Natl Acad Sci US A 91, 4579-4583.

Rasoloson, D., Tomková, E., Cammack, R., Kulda, J. \& Tachezy, J. (2001). Metronidazole-resistant strains of Trichomonas vaginalis display increased susceptibility to oxygen. Parasitology 123, 45-56.
Steinbüchel, A. \& Müller, M. (1986). Anaerobic pyruvate metabolism of Tritrichomonas foetus and Trichomonas vaginalis hydrogenosomes. Mol Biochem Parasitol 20, 57-65.

Swain, M. \& Ross, N. W. (1995). A silver stain protocol for proteins yielding high resolution and transparent background in sodium dodecyl sulfate-polyacrylamide gels. Electrophoresis 16, 948-951.

Tachezy, J., Kulda, J. \& Tomková, E. (1993). Aerobic resistance of Trichomonas vaginalis to metronidazole induced in vitro. Parasitology 106, 31-37.

Thong, K. W. \& Coombs, G. H. (1987). Comparative study of ferredoxin-linked and oxygen-metabolizing enzymes of trichomonads. Comp Biochem Physiol B 87, 637-641.

Vaňáčová, Š., Tachezy, J., Ullu, E. \& Tschudi, C. (2001a). Unusual diversity in $\alpha$-amanitin sensitivity of RNA polymerases in trichomonads. Mol Biochem Parasitol 115, 239-247.

Vaňáčová, Š., Rasoloson, D., Rázga, J., Hrdý, I., Kulda, J. \& Tachezy, J. (2001b). Iron-induced changes in pyruvate metabolism of Tritrichomonas foetus and involvement of iron in expression of hydrogenosomal proteins. Microbiology 147, 53-62.

Wang, A. L. \& Wang, C. C. (1985a). Isolation and characterization of DNA from Tritrichomonas foetus and Trichomonas vaginalis. Mol Biochem Parasitol 14, 323-335.

Wang, A. L. \& Wang, C. C. (1985b). A linear double-stranded RNA in Trichomonas vaginalis. J Biol Chem 260, 3697-3702.

Yarlett, N., Yarlett, N. C. \& Lloyd, D. (1986a). Metronidazoleresistant clinical isolates of Trichomonas vaginalis have lowered oxygen affinities. Mol Biochem Parasitol 19, 111-116.

Yarlett, N., Yarlett, N. C. \& Lloyd, D. (1986b). Ferredoxindependent reduction of nitroimidazole derivatives in drugresistant and susceptible strains of Trichomonas vaginalis. Biochem Pharmacol 35, 1703-1708.

Received 20 December 2001; revised 15 April 2002; accepted 2 May 2002. 